\title{
Explainable AI in Internet of Control System Distributed at Edge-Cloud Architecture
}

\author{
Mehdi Roopaei, Hunter Durian, Joey Godiska
}

\begin{abstract}
Many current control systems are restricted to highly controlled environments. In complicated dynamic and unstructured environments such as autonomous vehicles, control systems must be able to deal with more and more complex state situations. In complex systems with large number of states, it is often too slow to use optimal planners and developing heuristic tactics for high level goals can be challenging. AI control is an attractive alternative to traditional control architectures due to their capability to approximate optimal solutions in high dimensional state spaces without requiring a human-designed heuristic. Explainable AI control attempts to produce a human readable control command which is both interpretable and manipulable. This paper is an attempt to propose an architecture for explainable AI control in edge-cloud environment in which there are connected autonomous agents that need to be controlled. In this architecture the designed controller is distributed across the edge and cloud platform using explainable AI. This architecture could be introduced as Internet of Control Systems (IoCS), which could be applied as distributed tactics to control of connected autonomous agents. The IoCS attempts to unleash AI services using resources at the edge near the autonomous agents and make intelligent edge for dynamic, adaptive, and optimized AI control.
\end{abstract}

Keywords: AI Control, Explainable AI, Autonomous Agent, Edge-cloud architecture, Distributed Control Systems

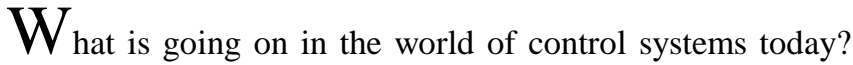
New methods of designing control systems are continuously being developed and engineered. One of the most common model-based methods. Model-based control systems are used in simple to complex systems [1]. Due to control systems becoming more complex, there is an increasing concern safe and reliable operation of control systems [2]. Even though there are many adaptive control techniques, they are not robust enough for many real-world problems where the degree of uncertainty is high which causes the methods of

Manuscript received on February 15, 2021.

Revised Manuscript received on February 22, 2021.

Manuscript published on February 28, 2021

* Correspondence Author

Mehdi Roopaei*, Electrical and Computer Engineering Department, university of Wisconsin-Platteville, Platteville, Wisconsin, USA. Email: roopaeim@uwplatt.edu

Hunter Durian, Electrical and Computer Engineering Department, university of Wisconsin-Platteville, Platteville, Wisconsin, USA. Email: durianh@uwplatt.edu

Joey Godiska, Electrical and Computer Engineering Department, university of Wisconsin-Platteville, Platteville, Wisconsin, USA. Email: godiskaj@uwplatt.edu

(C) The Authors. Published by Blue Eyes Intelligence Engineering and Sciences Publication (BEIESP). This is an open access article under the CC BY-NC-ND license (http://creativecommons.org/licenses/by-nc-nd/4.0/)

\section{INTRODUCTION} and oldest methods of designing control systems is using among the scientific community and industrialists over the

mathematical modeling and control to fail [2]. Data driven control is a new method that engineers are still trying to implement into control systems, but they have been able to develop some theories. Data driven control is the designing of a control system without using a model and instead it directly uses the input-output data of the system, a modeless control system [1]. Machine intelligence is also being incorporated into many systems because of the computer technology [2]. A major development in control systems is incorporating intelligence into the systems to reduce the complexities in the system [2]. Data driven control is used to get rid of the challenging issues of model-based theories and methods like robustness and modeling [1]. Some data driven control methods are cognitive control, neural-network-based control, and intelligent control [3]. Artificial Intelligence (AI) can be used in control to solve several difficulties that arise in the control systems. The hard control problems can be reopened to look for new solutions by integrating control science and data science together which is the main ideas of artificial intelligence [4]. How is using control systems and AI together beneficial? The combination of control systems technology and AI can help improve the quality and applicability of control, expanding out of the linear dynamic assumption [4]. For example, model-based dynamic optimization provides an optimal control solution with guaranteed stability and robustness, but the fine tuning of the model or switching to a global non-linear system can be a never-ending story in the case of performance issues [4]. Therefore, switching to the model-less Q-learning and direct optimization of control policy using real-time process data can be a more straightforward solution which can be turned into a generic algorithm [4]. When did artificial intelligence in control start and how has it evolved over the years? Artificial Intelligence in control started as a dream, like most inventions, for machines to have human like abilities like moving and decision making [5]. The actual research and development of implementing AI in control did not start until the mid-1900s [5]. The first ideas of AI in control started with three big conferences in the 1950s, but the first type of artificial intelligence, called Bayesian learning, was never fully developed until the 1980s [5]. Other types of AI in control to follow Bayesian learning are neural network control, machine learning control, reinforcement learning, neuro-fuzzy control, genetic control, and most recently, cognitive control [5]. In the 1980s, Bayesian learning was being invented. Bayesian learning does not use models. Bayesian learning uses probabilities and graphs [5]. Bayesian learning's primary role is computing probabilities of various nodes given the probability of others to construct graphs [5]. Bayesian networks are relatively easy to set up for both large and small networks [5].

Published By:

Blue Eyes Intelligence Engineering and Sciences Publication 
Bayesian learning is very important because the system can be automatically constructed from large databases [5]. Since the idea of Bayesian learning was invented, a branch of AI control called machine learning was created [5]. Machine learning has several different networks or methods that were invented [5].

The goal of machine learning is to construct hypotheses from data [5]. Some methods of machine learning are called memory-based learning, case-based reasoning, decision trees, neural networks, unsupervised learning, and reinforcement learning [5]. All these methods follow the same idea of machine learning but are done in a different mathematical or logical approach to obtain the control system [5]. Artificial intelligence in control has paved the way to new inventions and technology that have been created in the last few decades. These methods of AI control have been used to make robots and computer programs have human-like abilities [5]. For example, robots and computer programs have been created to beat humans who are professionals in videos games, chess, checkers, etc. [5]. Even appliances in homes are using the ideas of AI control to make them more intelligent [5]. Overall, AI control has just started and new developments like neural networks and cognitive control are still being developed and invented.

\section{MODEL-BASED CONTROL HISTORY}

How has model-based control evolved over the years? Model-based control has been around since the late 1940s and it is still one of the most common types of control design techniques. Model-based systems uses models to design controllers. Models are typical block diagrams. Model-based control has grown and developed into different branches that are used extensively in industrial processes, aerospace, traffic systems, and many other applications [3]. These branches are called adaptive control, system identification, robust control, optimal control, variable structure control, stochastic system theory, etc. [3]. Model-based control techniques are used in both linear and nonlinear systems. The linear system design methods include zero-pole assignment, LQR design, and robust control [3]. Nonlinear system design methods include Lyapunov-based controller designs, backstepping controller design, and feedback linearization, etc. [3].

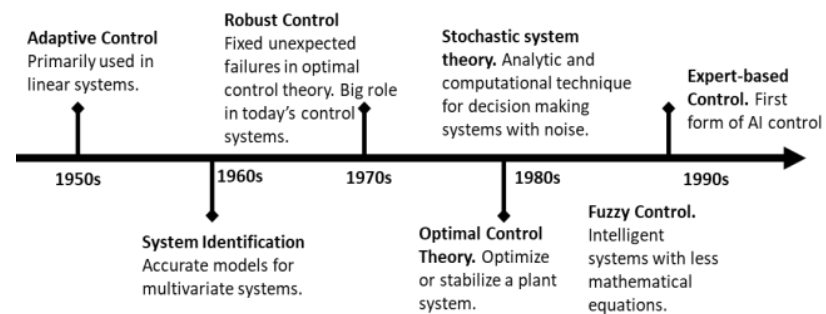

Fig. 1. A timeline of when the different model-based methods were researched and/or developed.

Adaptive control was theorized in the 1950s and developed since then to be used in several applications of model-based control [6]. Adaptive control is primarily used in linear systems [6]. It can be applied to many other applications, but it may need to ask for help from other methods, or a trial and error method is required [6]. Adaptive control has issues because the number of parameters that needs to be estimated is too large [6]. In other words, it is difficult to converge to a parameter because of inaccuracies, disturbances, and other reasons in engineering practices [6]. System Identification method was theorized and originated in the 1960s [7]. The advantage of system identification is the ability to provide accurate models for multivariate linear systems from the input-output data [7]. In the 1970s, a revolution in control theory developed when robust control was brought into the playbook [8]. The goal of robust control is to fix the unexpected failures of optimal control theory to produce feedback control designs that can tolerate the differences between design models and reality [8]. Robustness still plays a big role in control designs today and is also being used in data driven control theories [8]. Optimal control theory was researched in the 1980s [9]. The theory focuses on making optimal feedback logic more robust to variations in the plant and disturbance models [9]. The theory is used to optimize or stabilize a plant system. One element of optimal control theory is worst-case and $\mathrm{H}$-infinity control, which developed out of differential game theory [9]. Variable structure control was theorized from 1957-1970s, but it was not fully developed until the 1980s [10]. It is used in a wide range of control systems like nonlinear systems, multi-input/multi-output systems, discrete-time models, large-scale and infinite-dimensional systems, and stochastic systems [10]. The objective of variable structure control is to stabilize the system along with other control functions [10]. Variable structure control has the ability to create very robust control systems that can be completely insensitive to parametric uncertainty and external disturbances [10]. Another model-based control method called stochastic system control theory was researched in the 1980s and eventually becomes more developed. Stochastic control theory provides analytic and computational techniques for the decision making in stochastic systems with noise [11]. It is used for models like Markov decision making, linear quadratic, and Gaussian systems. Stochastic control relies on previous observations from the central system [11]. Expert-based control system was developed in the late 1980s and it is considered one of the first forms of artificial intelligence software [12]. The system is a computer system/code that produces decision making results like a human expert [13]. The system is primarily used to solve complex systems by using bodies of knowledge like if-then rules [13]. A type of expert-based control is called fuzzy control and was developed in the late 1980s. Fuzzy control makes the machines more intelligent to act more in a fuzzy manner like humans [14]. In other words, it helps with uncertainty, impreciseness, and qualitative decision-making problems [14]. Fuzzy control does not use a lot of mathematical equations. Making it user-friendly to develop control systems and helps the designers focus more on the functional objectives of the system [14]. Overall, there are several types of model-based control systems that were developed in the 1900s and are still used today. These control systems were revolutionary in improving and solving engineering problems for aerospace systems, traffic systems, manufacturing processes, and many other applications.

Published By:

Blue Eyes Intelligence Engineering and Sciences Publication

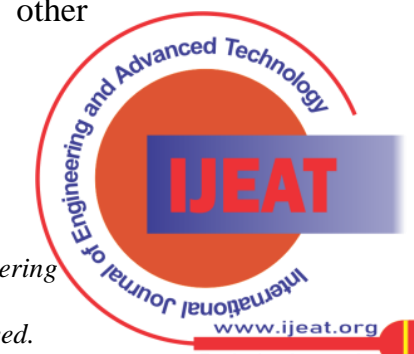


Even though the model-based control systems are still used today, they are very hard to implement in complex systems. So, new types of control systems are being developed.

The new types of control systems are defined as data driven control or modeless control, which already has several theorized systems like cognitive control, artificial intelligence control, neural networks, deep learning, and a couple other systems, figure 1 .

\section{ARTIFICIAL INTELLIGENCE IN CONTROL}

Artificial Intelligence can be quickly summarized as the display of intelligent behavior normally exhibited by humans, implemented by a computing system, most commonly in the context of decision making [15]. Why use artificial intelligence in control? The driving motivation behind $\mathrm{AI}$ is to make some decision that achieves a desirable outcome. Artificial intelligence is desirable in a control system environment because of the characteristics of autonomous systems. The use of AI in control is beneficial in several ways. AI helps to understand complex systems that are arising in control systems [16]. It can be used to solve new approaches to those complex problems because it can be easily organized and synthesized [16]. Artificial intelligence can also solve problems themselves [16]. AI is used to solve control problems by using algorithmic approaches [16]. This allows for desirable implementation characteristics [16]. For example, autonomous control systems are typically very complex, and it is either impossible or inappropriate to use differential equations or difference equations to represent the system [16]. It may be inappropriate to use the differential equations or difference equations because it makes the subsequent analysis of the system difficult, therefore the use of AI in control is used [16]. So, using AI in control can make the subsequent analysis easier given that the specifications are not highly demanding [16]. The use of AI in control must be inherent. It should prevent failures that could ruin the system's mission goals or safety [16]. So, using AI in control can create a self-testing system to achieve system safety and the mission goals [16]. The design of the AI controller must protect the system from erroneous or valid external commands, avoid irreversible state changes, and have adjustable fault detection thresholds [16].

What are some guidelines for the controller design of autonomous systems that allow AI to be beneficial for their control? The basic structure of the autonomous control system must be hierarchical, lower-level subsystems still need to take some actions to enhance control systems, they need to clear it with a higher-level authority before any execution [16]. The use of AI control helps to provide intelligent communication between the higher levels and lower levels of the autonomous control system and causes it to utilize fewer conventional numeric-algorithmic methods and more symbolic-decision making methods [16]. Besides, the autonomous control system must be visible or transparent so the human in control loop have ultimate supervisory control on functionality of any sub-systems when necessary [16]. The explainable AI helps the human to have more visibility on any function or operation at any level of control. In the next session we have a review on the three main AI architectures preciously invented for the control of autonomous agents.

\section{A. Artificial Intelligence-Command and Control Systems}

In [17], a Decision Support in Command and Control Systems was developed which is an important and complex application of AI is in the field of military Command and Control (C2). In C2, the central problems - whether their solutions are classical or AI-based - come from three questions [18]:

- How can one obtain a collective effect from a large set of resources?

- How can one handle inherent uncertainties?

- How can one produce an impact at a faster pace than the adversarial networks?

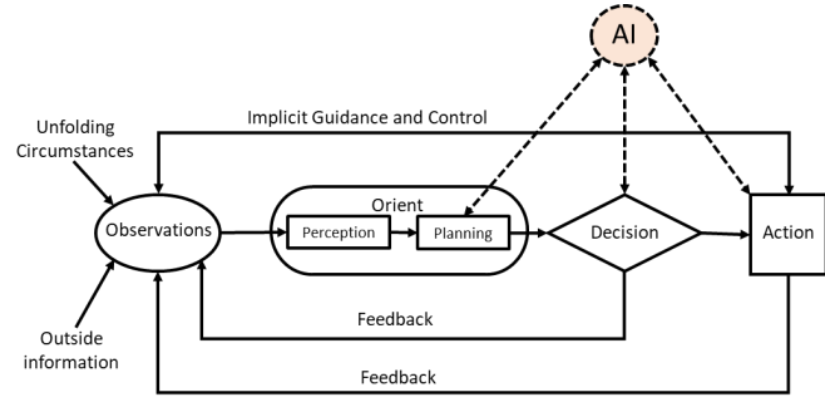

Fig. 2. The simple DOODA-loop is expanded to account for more detailed information proposed in [19]. The authors modified their method and add AI in [17]

The effectiveness of how these questions are answered depends on the effectiveness of the C2 model implemented. As of 2005 and through today, the dominant model of C2 is the OODA (Observe-Orient-Decide-Act) loop developed by Boyd in 1987 [19]

In the proposed model, Observing involved noting the characteristics of the environment, Orienting involved positioning oneself in an advantageous way, Deciding has to do with how the last stage, Acting, is conducted, and after an action is taken, the loop restarts. Given that this was developed in a military context, arriving at a clear outcome of winning over losing was the purpose of the OODA-loop, an outcome that would have to evolve to become more multi-faceted, figure 2 .

Boyd's model could be expanded to situations beyond fighter jets, and thus an updated model was needed [19]. As seen in figure 2, the OODA-loop was expanded into a multi-stage model that detailed the interactions between each stage of the loop. In the implementation of the OODA loop, there is feedback from the hypothesis, from the action as well as its effects on the environment, from outside information, and from changing circumstances. Given the intricate nature of this model, the system should be continuously updating. The OODA-loop is transformed to a Dynamic Orient, Observe, Decide, and Act loop, or a DOODA-loop. This loop iterates through its steps until a conclusion is reached, accomplishment or failure. Given the added complexity brought on by creating a dynamical system combined with the large amount of sensor data,

\section{Published By:}

Blue Eyes Intelligence Engineering and Sciences Publication (C) Copyright: All rights reserved.

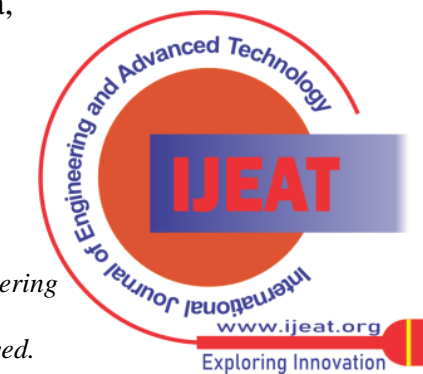


an Artificial Intelligence system would be beneficial [17].

Three different tasks were identified in [17] where AI was identified to be the most useful: analysis and monitoring, planning, and execution. Within the analysis group, finding and compiling information as well as anomaly detection were named as important sub-tasks. In analysis and monitoring, gathering information is a large-scale operation that can be automated using AI techniques to find, sort, and compile information. The subtask of anomaly detection in analysis is done by the use of deep autoencoders (a machine learning system that simplifies high-dimensional information and reconstructions low-dimensional information) to detect residuals between "normal" data points and outliers.

\section{B. Artificial Intelligence-Planning}

A key element within an Artificial Intelligence system is the planning stage of the loop. After integrating and interpreting the data, the planning stage comes prior to any executive functions of the process at hand. Compared to a classical control system, the planning phase is analogous to the physical plant [20]. The goal of the planning system is to achieve the desirable output while reducing any error between the actual and desired output. This goal necessitates a feedback loop, as seen in figure 3. The concept of the AI planner comes from [20], where the components, functions, and characteristics are defined. Several functional components exist within the AI planning system. Firstly, initial plans must be synthesized for achieving the goal at hand. These plans can be re-synthesized or re-planned if there is any sort of failure in achieving the goal. After this plan is generated, the outcome is simulated, or projected, to attempt to determine if the generated plan is suitable. Again, this process can be iterated until a desired value is achieved. Plan decision rules determine which generated plan should be carried to the executive portion of the system, where the plans are translated into an actionable format. After the plan is executed, several things occur that assist the system in generating the next plan. The effects on the problem domain/environment are measured and assessed, as well as the success of plan execution. It is noted that in the "Situation Assessor" block, those particular terms are chosen due to the nonconforming nature of system's state and the lack of hard, fast, definitions on that state [20].

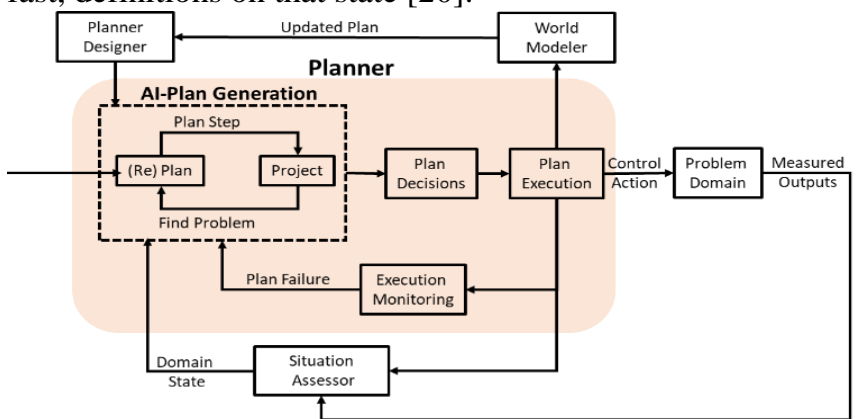

Fig. 3. The AI planning system will identify changes in the system and update the problem-solving algorithm automatically. This is another iterative process that optimizes the plan generation process within the OODA-loop.

\section{Artificial Intelligence Cognitive}

Cognitive Control is an emerging control system that is based off the human brain control system. In human psychology, performance in cognitive tasks is defined as “. . . two distinct processes: acquisition of knowledge and cognitive control over knowledge already acquired" [21]. Human behavior is inherently very complex but can be boiled down to a few simple statements. Other definitions discuss coordination of thoughts, actions, and goals, or evaluation of outcomes as a result of actions. In short, human cognitive control, as with all control systems, can be simplified as the manipulation of the input data to achieve a desired output the perception-action cycle [21]. Another useful understanding in human cognitive control and cognitive control in an engineering context is the information gap. When receiving data, several processes occur. Initially, the relevant information is extracted from the noisy and redundant information. The information gap is the information that that is necessary to complete the task but is not present in the relevant information. In other words, the information gap is the difference between the present information that pertains to the problem at hand and enough information. In the paper "Cognitive Control", the information gap is metrified into a parameter known as entropic state [21]. From Joaquin Fuster, a Spanish neuroscientist, and his book, "Cortex and Mind: Unifying Cognition" [22], Fatemi and Haykin [23] derived this model of a Cognitive Dynamic System (CDS) shown in figure 4. In this model, the executive and planning parts of the model are on the left- and right-hand sides, respectively. Analogous to the cognitive system of a human, the input to the executive functions of the CDS does not come directly from the outside world, and instead must pass through the perceptual part of the system.

\section{EXPLAINABLE AI CONTROL}

There are several challenges in current AI domain. One concern is how to regulate an AI network when utilized as a problem-solving platform. AI is not restricted by traditional ways of thinking or solving a problem, since it tackles an issue with brute force and such a high number of iterations, whereas a human will base their solution largely off of prior knowledge, with less lateral thinking than an AI system. Because of this, the designers of an AI system cannot predict or control what it will do when making autonomous decisions [24]. Another issue in Artificial Intelligence discipline is regulation of these intelligent networks. Two types of control loss are described as local loss of control, and general loss of control. The former is when the legal supervisory human for the AI system can no longer control the system, and the latter is when no human can control the system [24]. Clearly, one of these situations is more threatening than the other. A necessary part of designing an AI system is defining its limits, i.e. when enough is enough. Therefore, there is a need for a kind of AI network which could be interpretable and predictable Explainable Artificial Intelligence has these characteristics.

Published By:

Blue Eyes Intelligence Engineering and Sciences Publication

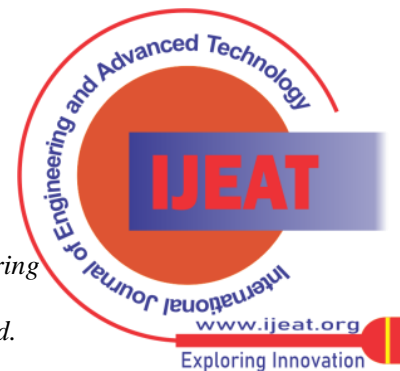


Cognitive Control

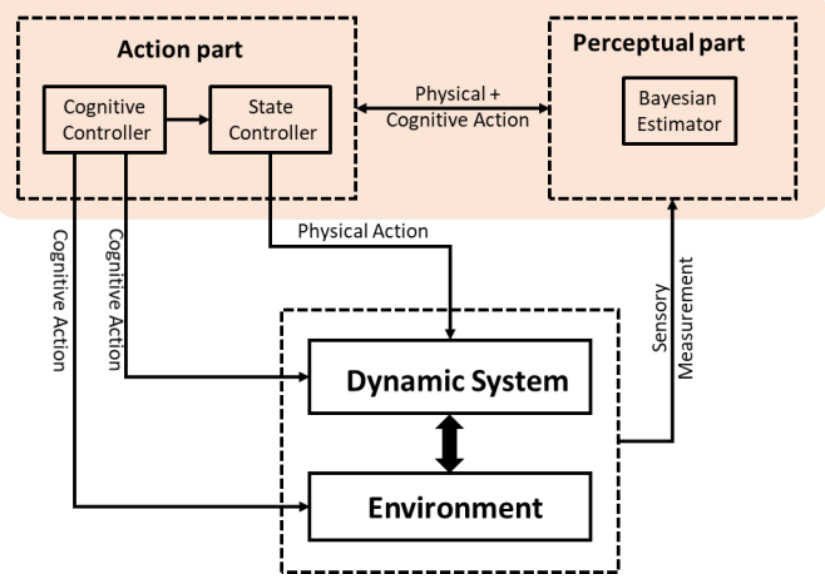

Fig. 4. The Cognitive Control system as developed in [23] shows a simple model of the control process in the human brain. Note that the Action part of the system does not directly receive information from the environment and must receive information from the perceptual side first.

Although explainable artificial intelligence has been a recent discovery, it is not a new concept nor new in artificial intelligence or machine learning [25]. The use of explainable artificial intelligence has come about in recent years due to the methods of deep learning not being interpretable [25]. Using symbolic regression to make the models has be used for a long time which is very explainable [25]. For example, genetic programming was used for network control because it is extremely general [25]. However, the generalized regression methods are not flexible or explainable [25]. Now, models of machine learning are primarily focused on the subsequent analysis of the analytical expressions [25]. Finding control laws and/or mathematical models are beneficial to obtain an explainable model of an artificial intelligent control system [25]. The models then can be used for a stability analysis of the system and obtain other requirements of the system [25]. Overall, explainable artificial intelligence is beneficial because mathematical models can be developed to interpret the control systems, which can then be automatized to do a fast analysis of the system [25].

\section{A. Proposed Architecture for Internet of Control System}

The proposed architecture in this paper attempts to control the connected autonomous agents using distributed AI control at edge to cloud level, called Internet of Control Systems (IoCS). IoCS employs AI analytics at edge level to provide real-time control of autonomous agents at end level. However, we need to check the possibility of providing AI services at edge level. The AI models become deeper and require larger-scale dataset to improve their accuracy. Undoubtedly, it is difficult to deploy the AI models in the edge with limited resources. Traditionally, those intelligent models are generally deployed in the cloud however, the cloud analytics limits the ubiquitous distribution of $\mathrm{AI}$ services. Precisely, it cannot guarantee the delay requirement of real-time services, e.g., real-time control with strict latency demands. Furthermore, for critical data sources, data safety and privacy protection should be addressed in cloud analytics. To take care of these issues, AI services should be injected to edge analytics and should be further adapted to fit in the resource-constrained edge, while wisely treating the trade-off between the planning and decision accuracy and the action latency of them. Providing distribution of AI control at edge requires the support of edge analytics. This support is not just at the control architecture level, any edge hardware and software are equally significant, and they need to be reinforced in design, adaptation, and optimization. AI can learn to handle decision-making problems by interacting with the environment. The capabilities of edge devices in analytics and storage, along with the collaboration of the cloud, make it possible to use AI to provide intelligent edge control by optimizing edge computing networks and systems. Therefore, the proposed IoCS can be applied to learn the long-term optimal resource management and task scheduling strategies, to achieve the intelligent control of the edge. Edge analytics requires the distribution of AI models for training because of lacking computational and memory resources in autonomous agent and/or end devised at the field. The distribution process splits a large AI model into several sub-models and then handles the training procedure for the segmented models in parallel. The training speed can be improved using parallel processing; however, this process is required for the situation that the AI model is larger than the device/agent memory. Training a large AI model needs a lot of computation resources; thousands of CPUs are required to train a large-scale AI model. In order to solve this issue, distributed analytics at IoT, Edge, and Cloud can be utilized for model parallel training [26]. In a scenario where many end devices, edge nodes, and cloud data centers are scattered and envisioned to be connected by virtue of edge computing networks, the training of AI models should be handled by the cloud analytics, figure 5. At the cloud level, the cloud is in charge of integrating the AI models among the edge nodes and updating parameters of distributed AI models on edge level [27]. Since the distributed AI model on an edge node might be significantly diminished due to its local knowledge, the cloud needs to integrate various AI models, which have enough training to accomplish global knowledge. When the edge is unable to deliver the service confidently, the cloud can use its powerful computing power and global knowledge for supplementary processing and support the edge nodes to bring the updated AI models. To obtain the best trade-off between model accuracy and processing delay, multiple AI models with different model performance can be preserved for each AI service. Then, by intelligently choosing the best model, the desired adaptive AI model for control is attained [28].

\section{CONCLUSION}

In this paper an architecture for explainable and distributed AI control in edge-cloud architecture is proposed.

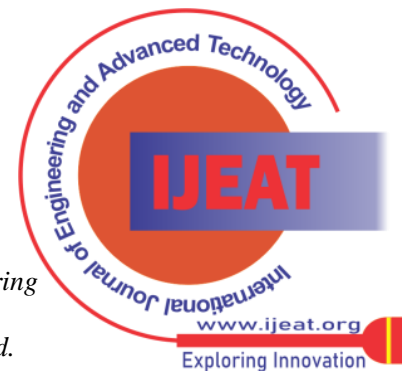




\section{Explainable AI in Internet of Control System Distributed at Edge-Cloud Architecture}

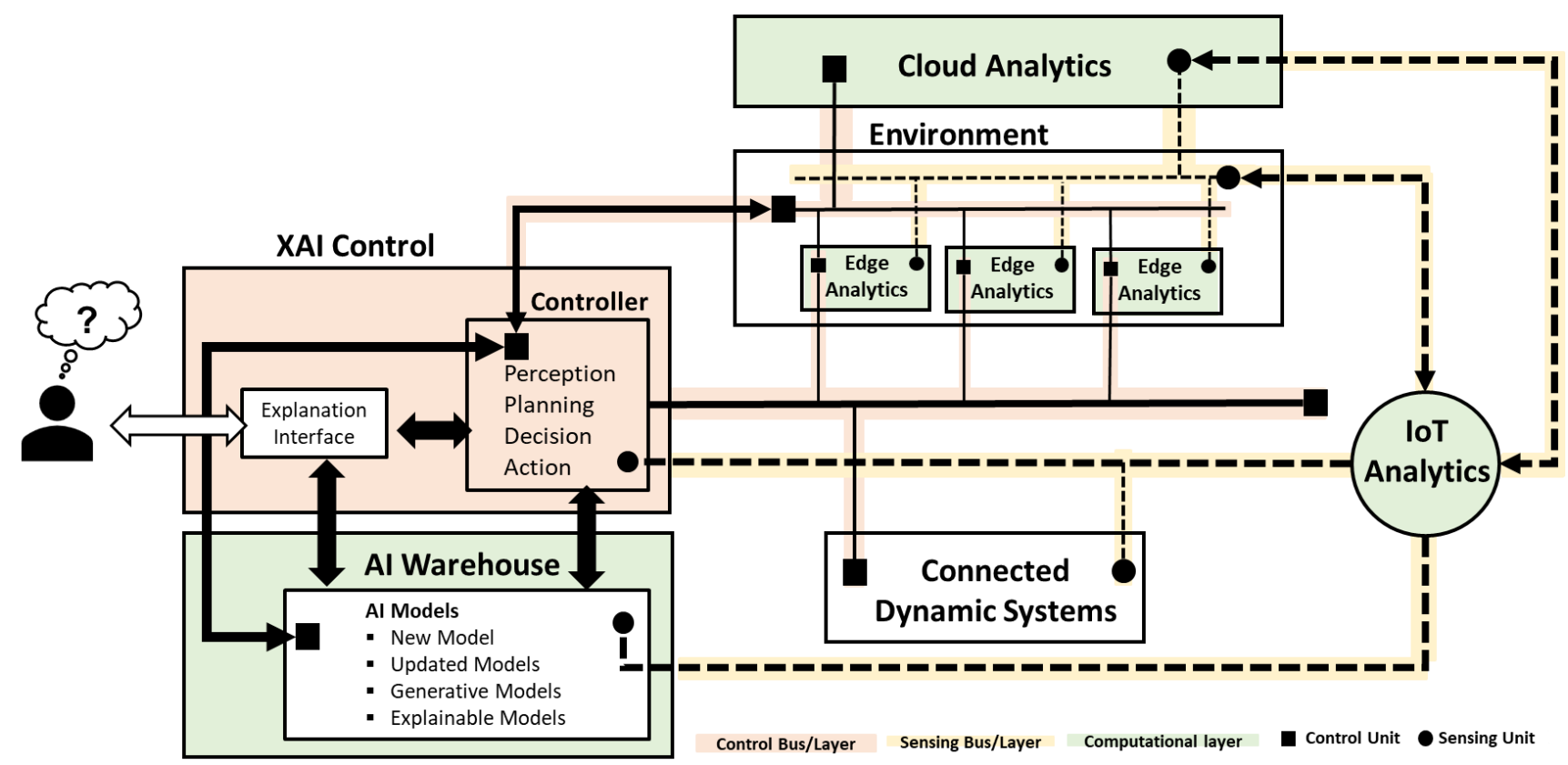

Fig. 5. The architecture of Internet of Control Systems (IoCS). The explainable AI let the user interacts with the control layer, where processes within the system are relayed to the user in a practical and understandable format. Environmental data is collected by the Internet of Things, then stored and analyzed in the edge and cloud layers.

The new control architecture attempts to control connected autonomous agents at field level utilizing IoT devices at edge and cloud level. The proposed architecture is called: Internet of Control Systems (IoCS) which is distributed across the edge and cloud platform using explainable AI. The IoCS provides AI services using resources at the edge near the autonomous agents and makes intelligent edge for dynamic, adaptive, and optimized AI control. Employing IoT analytics, edge AI-controllers combine sensing and control of agents and communicating with an array of data producers, transform their data in meaningful ways, and deliver it securely to decision-making control units. Explainable AI provides visibility into how an AI-Control system makes decisions and predictions and accomplishes its actions. Explainable AI-Control describes the rationale for the decision-making process and delivers a sense of how the control system will behave in the future.

\section{REFERENCE}

1. Z. Hou, H. Gao and F. Lewis, "Data-Driven Control and Learning systems," IEEE Transactions on Industrial Electronics, vol. 64, no. 5, pp. 4070-4075, 2017.

2. N. M. V. Kumar, "The application of Artificial Intelligence Techniques for Intelligent Control of Dynamical Physical Systems", International Journal of Adaptive Control and Signal Processing, Vol. 8, pp. 379-392, 1994.

3. Z. Hou and Z. Wang, "From Model-Based Control to Data-Driven Control: Survey, Classification, and Perspective," Science Direct Information Sciences, vol. 235, pp. 3-25, 2013.

4. V. Hallena, "Control and Optimization Algorithms Based on Artifical Intelligence/Machine Learning Approaches," Faculty of Electrical Engineering CTU in Prague, [Online]. Available: https://dce.fel.cvut.cz/en/control-and-optimization-algorithms-basedartificial-intelligence-machine-learning-approaches. [Accessed 2312 2020].

5. N. Nilsson, "The Quest for Artificial Intelligence," Cambridge University, 2009, pp. 0-70.

6. J. Hu, H. Wu and Y. Xie, "Characteristic Model-Based All-Coefficient Adaptive Control Method and Its Applications", IEEE Transactions on Systems, Man, and Cybermetics, Part C, Volume 37, Issue 2, pp. 213-221, 2007.
7. M. Viberg, "Subspace-based Methods for the Identification of Linear Time-invariant Systems", Automatica, Volume 31, Issue 12, pp. 1835-1851, 1995.

8. M. Safonov, "Orgins of Robust Control: Early History and Furture Speculations", Anual Reviews in Control, Volume 36, Issue 2, pp. 173-181, 2012.

9. E. B. Jr., "Optimal Control-1950-1985", In IEEE Control Systems Magazines, Volume 16, Issue 3, pp. 26-33, 1996.

10. W. Gao, J. Y. Hung and J. C. Hung, "Variable Structure Control: A Survey", IEEE Transactions On Industrial Electronics, Volume 40, Issue 1, pp. 2-22, 1993.

11. A. Nayyar, A. Mahajan and D. Teneketzis, "Decentralized Stochastic Control with Partial History Sharing: A Common Information Approach", IEEE Transactions on Automatic Control, Volume 58. Issue 7, pp. 1644-1658, 2013.

12. S. J. R. Peter Norvig, "Artificial Intelligence: A Modern Approach", Prentice Hall, 1994, p. 1037.

13. P. Jackson, "Introduction to Expert Systems," Addison Wesley, pp. 2, 1998.

14. D. Drainkov, H. Hellendoorn and M. Reinfrank, "An Introduction to Fuzzy Control", Springer-Verlag Berlin Heidelberg, pp. 1-5, 1993.

15. M. A. D. Pramod P. Khargonekar, "Advancing systems and Control Research in the Era of ML and AI," Annual Review in control, vol. 45, pp. 1-4, 2018.

16. P. Antsaklis, K. M. Passino and S. Wang, "An Introduciton to Autonomous Control Systems," in Fifth IEEE International Symposium on Intelligent Control, Philadelphia, 1991.

17. J. Schubert, J. Brynielsson, M. Nilsson and P. Svenmarck, "Artificial Intelligence for Decision Support in Command and Control Systems," in 23rd International Command and Control Research \& Technology Symposium, Pensacola, 2018.

18. B. Brehmer, "Command and Control as Design," in Proceedings of the 15th International Command and Control Research and Technology Symposium, Washington DC, 2010.

19. B. Brehmer, "The Dynamic OODA Loop: Amalgamating Boyd's OODA Loop and the Cybernetic Approach to Command and Control," in 10th International Command and Control Research and Technology Symposium The Future of C2, McLean, 2005.

Published By: 
20. K. M. Passino and P. J. Antsaklis, "A system and control theoretic perspective on artificial intelligence planning systems.," Applied Artificial Intelligence an International Journal, vol. 3, no. 1, pp. 1-32, 1989.

21. S. Haykin, M. Fatemi, P. Setoodeh and Y. Xue, "Cognitive Control," Proceedings of the IEEE, vol. 100, no. 12, pp. 3159-3169, 2012.

22. J. Fuster, "Cortex and Mind: Unifying Cognition", Oxford University Press, 2002.

23. M. Fatemi and S. Haykin, "Cognitive Control: Theory and Applications," IEEE Access, vol. 2, pp. 698-710, 2014.

24. M. U. Scherer, "Regulating artificial intelligence systems: risks, challenges, competencies, and strategies," Harvard Journal of Law \& Technology, vol. 29, no. 2, 2016

25. T. I. M. A. M. Quade, "Explainable Machine Learning Control-Robust Control and Stability Analysis," researchgate.net, January 2020.

26. A. Coats, B. Huval, T. Wang and e. al, "Deep learning with cots hpc systems," in 20th International Conference on Machine Learning, 2013.

27. J. Ren, Y. Guo, D. Zhang and e. al, "Distributed and Efficient Object Detection in Edge Computing: Challenges and Solutions," IEEE Network, vol. 32, no. 6, pp. 137-143, 2018.

28. S. S. Ogden and T. Guo, "MODI: Mobile Deep Inference Made Efficient by Edge Computing," in USENIX Workshop on Hot Topics in Edge Computing, 2018.

29. J. Virués-Ortega, "The case against B. F. Skinner 45 years later: An encounter with N. Chomsky.," BEHAV ANALYST, vol. 29, pp. 243-251, 2003.

30. D. Faggella, "Everyday Examples of Artificial Intelligence and Machine Learning," Emerj, 11 April 2020. [Online]. Available: https://emerj.com/ai-sector-overviews/everyday-examples-of-ai/. [Accessed 4 November 2020].

31. J. Danaher, "Is Regulation of Artificial Intelligence Possible?" hplusmagazine, 15 July 2015. [Online]. Available: https://hplusmagazine.com/2015/07/15/is-regulation-of-artificial-intel ligence-possible/. [Accessed 11 November 2020].

32. J. Akst, "A Primer: Artificial Intelligence Versus Neural Networks," The Scientist, 30th April 2019. [Online]. Available: https://www.the-scientist.com/magazine-issue/artificial-intelligence-v ersus-neural-networks-65802. [Accessed 11 November 2020].

33. A. Krogh, "What Are Artificial Neural Networks?" Nature Biotechnology, vol. 26, no. 2, pp. 195-197, 2008.

34. M. M. Y. R. Muhammad Waseem Ahmad, "Trees vs Neurons: Comparison between random forest and ANN for high-resolution prediction of building energy consumption," BRE Centre for Sustainable Engineering, vol. 147, pp. 77-89, 2017.

35. J. Ren, D. Zhang, S. He, Y. Zhang and T. Li, "A Survey on End-Edge-Cloud Orchestrated Network Computing Paradigms: Transparent Computing, Mobile Edge Computing, Fog Computing, and Cloudlet," ACM Computing surveys, vol. 52, no. 6, pp. 1-36 2020.

36. A. Bazanella, L. Campestrini and D. Eckhard, "Data Driven controller design: the H2 approach", Springer, 2012.

\section{AUTHORS PROFILE}

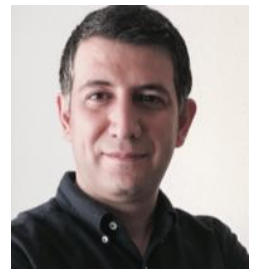

Mehdi Roopaei (M'02-SM'12) has a recognized record of contributions in intelligent control of dynamic systems. Roopaei, senior member of Institute of Electrical and Electronics Engineers (IEEE), received his B.S. and M.S. degree from Shiraz University, Department of Electrical Engineering in major of Control Systems in 2002 and 2006 respectively. He received Ph.D. degree in Computer Engineering from Shiraz University on adaptive fuzzy control of nonlinear systems in 2011. He was Post-Doctoral Fellow at University of Texas at San Antonio from 2012 to Summer 2018 and holds the title of Assistant Professor at the Department of Electrical and Computer Engineering in University of Wisconsin-Platteville on Fall 2018. His research interests include: AI-Driven Control Systems, Learning Dynamical System Models from Data, Machine Learning and Internet of Things (IoT), and Immersive Analytics. He is Associate Editor of IEEE Access and Editorial Board of IoT Elsevier journal. He is guest editor for special issue, IoT Analytics for Data Streams, at IoT Elsevier and published a book on "Applied Cloud Deep Semantic Recognition: Advanced Anomaly

Published By:

Blue Eyes Intelligence Engineering and Sciences Publication

Detection:”, CRC-Press, 2018. He was IEEE chapter chair officer for joint communication and signal processing communities at San Antonio, Jan-July 2018. He has over 60 peer reviewed technical publications, serves as program committee in several conferences and being as technical reviewer in many journals.

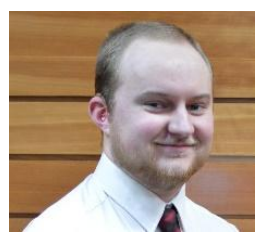

Hunter Durian is completing his senior year of study at the University of Wisconsin-Platteville, pursuing a bachelor's degree in electrical engineering. His emphasis of study is in Power and Controls which entails the topics of power transmission systems and modern control systems. His research interests include the rise in design and usage of Artificial Intelligence driven control systems, as well as how modern control systems are being developed used, and lastly how can control systems be used in Power transmission systems. This paper "Explainable AI in Internet of Control System

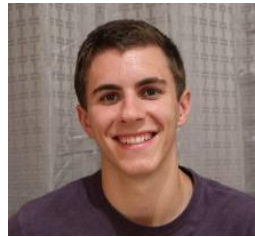

Joey Godiska is a completing his Senior year of study at University of Wisconsin-Platteville, pursuing a Bachelor of Science in Engineering Physics. His emphasis entails topics in classical and modern control theory, however, his major covers topics from mechanical engineering to quantum mechanics. While this paper, "Explainable AI in Internet of Control System Distributed at Edge-Cloud Architecture" is his first foray into academic publication, he has implemented his education for other extracurricular projects that include digital circuit design, low voltage analog circuit design, and microcontroller-based control systems. Currently, he focuses on furthering his studies on the implementation of IoT and its potential applications and limitations. 